\title{
2019 MRS Fall Meeting featured a variety of outreach, career, and professional development offerings
}

\author{
mrs.org/fall2019
}

$\mathrm{M}$ ore than 6600 people attended the 2019 Materials Research Society (MRS) Fall Meeting and Exhibit held in Boston from December 1 to 6 . The Meeting Chairs, Bryan D. Huey (University of Connecticut), Stéphanie P. Lacour (École Polytechnique Fédérale de Lausanne), Conal E. Murray (IBM T.J. Watson Research Center), Jeffrey B. Neaton (University of California, Berkeley, and Lawrence Berkeley National Laboratory), and Iris Visoly-Fisher (BenGurion University of the Negev), compiled 54 symposia and organized them into eight topical areas: Broader Impact; Electronic, Photonic and Magnetic Materials; Energy and Environment; Fabrication of Functional Materials and Nanomaterials; Materials for Quantum Technology; Materials Theory, Computation and Characterization; Mechanical
Behavior and Structural Materials; and Soft Materials and Biomaterials.

To complement these sessions, tutorials were offered in several technical areas, more than 227 exhibitors occupying 275 booths were on-site, and poster sessions were held during the evenings. An international exhibit showcased products and services of interest to the materials community. In addition, several special events highlighted science outreach.

The Public Outreach Committee featured a program on "The Role of Plastics in Achieving Energy Sustainability by 2050 -Friend or Foe?" Educators from The Pennsylvania State University's Materials Research Science and Engineering Center presented educational hands-on activities designed to help inform and educate young children and the public about materials science and sustainability and their personal relevancy in everyday life.

The National Science Foundation offered Speed Coaching with the Division of Materials Research. Program directors were available for one-on-one conversations on topics such as funding, future program ideas, and program relevance.

The iMatSci Innovation Showcase featured a Pitch Session Boot Camp for iMatSci startups; Startup Support Communities for Materials-Based Companies, which was a panel that discussed the different roles that various entrepreneurial support networks play in helping to get early-stage innovators started; a workshop on Creating an Effective Intellectual Property Strategy; and an Innovator Networking and Awards Reception.

The Science in Video Competition accepted submissions of two-minute

\section{MRS Fall Meeting Graduate Student Award winners}

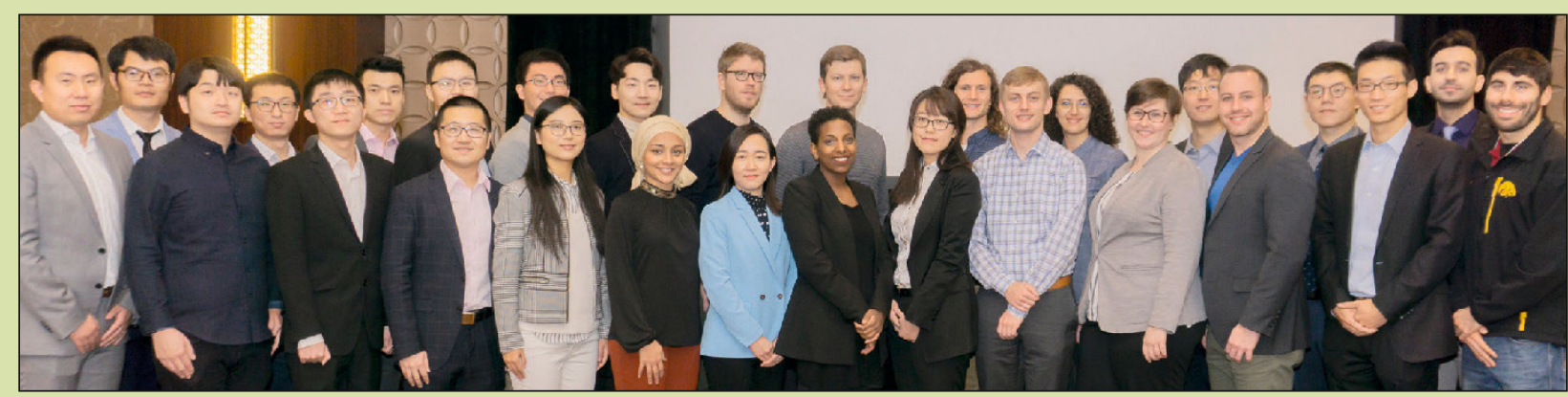

Front Row: Jia Zhuang, University of California, San Diego; Hua Zhu, Brown University; Minliang Lai, University of California, Berkeley; Yiren Zhong, Yale University; Xiaotong Li, Northwestern University; Naila Al Hasan, University of Maryland; Chengcheng Fang, University of California, San Diego; Lucie Ries*, Université de Montpellier; Juyoung Leem**, University of Illinois at Urbana-Champaign; David Russell Barton, Stanford University; Megan O. Hill, Northwestern University; Matthias T. Agne*, Northwestern University; Junzhe Lou, Stanford University; Nicholas Rolston, Stanford University. Back Row: Xuezeng Lu, Northwestern University; Yongtao Liu, The University of Tennessee; Jieun Kim, University of California, Berkeley; Yin Liu*, University of California, Berkeley; Tatsuya Higaki, Carnegie Mellon University; Yoonho Kim*, Massachusetts Institute of Technology; Martin H Dehn, The University of British Columbia; Andrey Vyatskikh*, California Institute of Technology; David Mackanic*, Stanford University; Ilaria Abdel Aziz*, Istituto Italiano di Tecnologia, Politecnico di Milano; Zhijie Zhu*, University of Minnesota Twin Cities; Chunhui Dai, University of Minnesota Twin Cities; Amirali Nojoomi, The University of Texas at Arlington. Missing: William Fitzhugh, Harvard University; Arda Kotikian, Harvard University; Siddharth Krishnan, University of Illinois at Urbana-Champaign; Peter Benjamin Meisenheimer, University of Michigan; Jaba Mitra, University of Illinois at Urbana-Champaign; Yan Wang*, University of Cambridge. 
videos about a wide range of materials science topics that were intended to teach and amuse a broad audience. The finalist videos were posted to YouTube, where viewers were able to vote for their favorite submission for the People's Choice Award. Babak Anasori, Indiana University-Purdue University, created and organized the competition, and sponsors included the MRS Foundation, Goodfellow Corporation, MilliporeSigma, and A.J. Drexel Nanomaterials Institute. The videos have so far received more than 2000 views online.

The MRS Fall Meeting Career Fair provided career development sessions; resume critiques, mock interviews, and professional photos; recruiters and on-site interviews; and job seeker and employer networking.

At the Materials Voice Booth, US attendees sent personalized letters to their representatives on Capitol Hill. Other Advocacy offerings included a session on the "Congressional Science and Engineering Fellowship Program," where attendees learned about MRS/ OSA and MRS/TMS Congressional Fellowships, and sessions on Research Funding Opportunities, which provided interaction between government agency presenters and MRS members.

The Professional Development program featured a Women's Workshop on "Understanding Unconscious Bias in the Workplace;" a session on the "Essentials of Getting Your Work Published," which offered "how to" advice to early-career researchers looking to polish their publishing skills; a "Careers at Government Laboratories and User Facilities" panel discussion; and the "United Nations Sustainable Development Goals Workshop-Agents for Sustainable Community Change," where participants learned about sustainability and how to align in the development of community action plans.

The Fall Meeting also featured the popular PowerPoint ${ }^{\text {TM }}$ Karaoke, where presenters explain a slide without prior knowledge of its content or author. Afterward, the MRS Frontiers Reception-Building Communities offered an opportunity to forge new communities on frontier topics in materials research: artificial intelligence, biomaterials, quantum materials, sustainability, and responsive and adaptive materials.

The MRS Awards and Recognition Program strives to acknowledge outstanding contributors to the progress of materials research and to recognize their accomplishments. At the Fall Meeting, MRS recognized Jerry D. Tersoff, IBM T.J. Watson Research Center, with the Von Hippel Award for advancing the understanding of low-dimensional and nanoscale electronic materials. Paula T. Hammond, Massachusetts Institute of Technology, was selected as the David Turnbull Lecturer for her contributions to the science, engineering, and applications of self-assembled macromolecular systems. Lu Sham, University of California, San Diego, received the Materials Theory Award for pioneering contributions to the quantum theory of molecules and solids, especially the Kohn-Sham formulation of density functional theory. Catherine J. Murphy, University of Illinois at Urbana-Champaign, and Haimei Zheng, Lawrence Berkeley National Laboratory, were selected as MRS Medalists for outstanding contributions on the study of anisotropic nanoscale materials, transformation, and application.

Kelsey Hatzell, Vanderbilt University, received the MRS Nelson "Buck" Robinson Science and Technology Award for Renewable

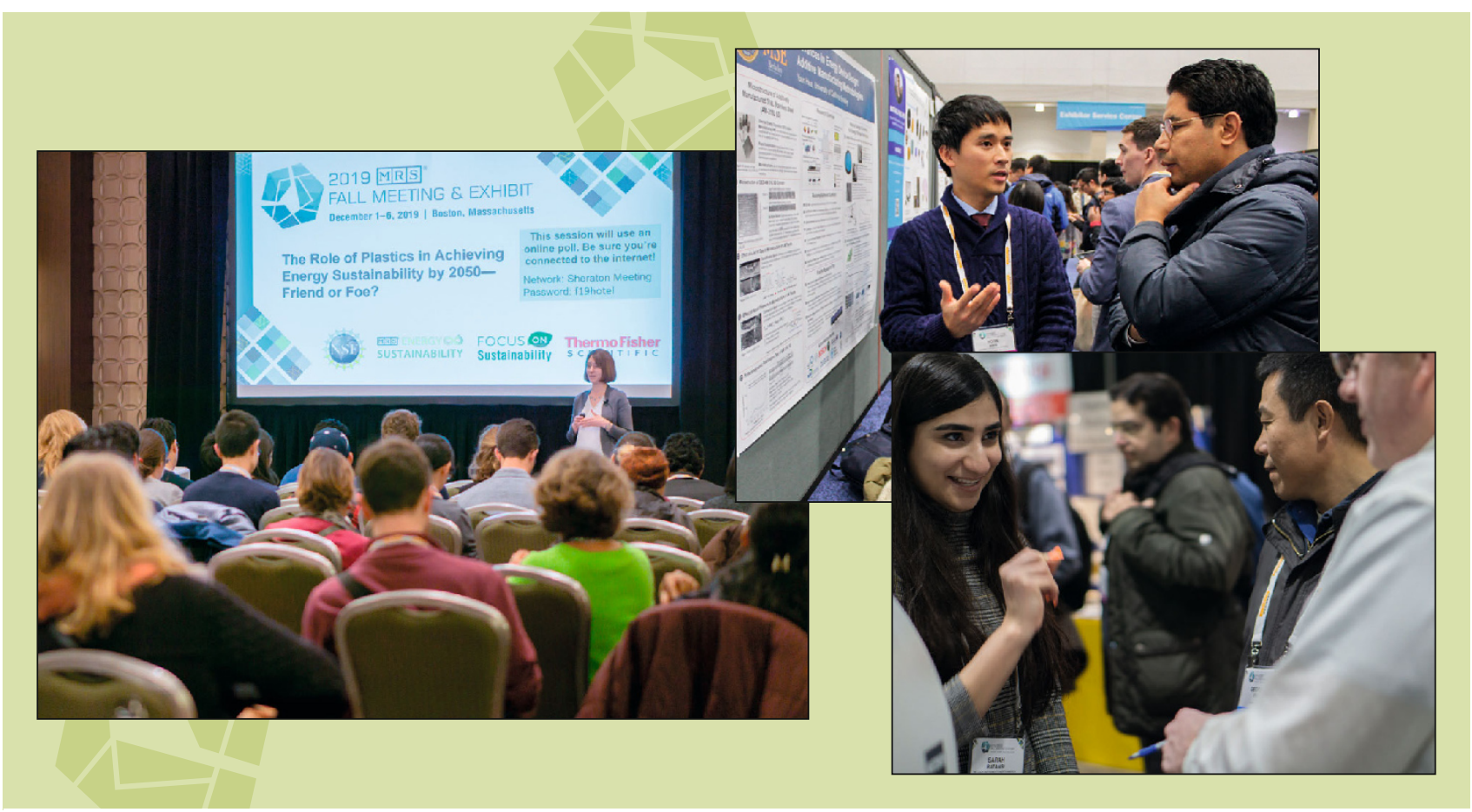


Energy for her group's work on multifunctional coatings and understanding phenomena at solid-liquid and solidsolid interfaces. Ognjen Ilic, University of Minnesota, received the MRS Bulletin Postdoctoral Publication Prize for his outstanding academic achievements, high quality of scientific publications, and demonstrated passion for science communication.

Sharon C. Glotzer, University of Michigan, presented The Fred Kavli Distinguished Lectureship in Materials Science, and Silvia Vignolini, University of Cambridge, presented The Kavli Foundation Early Career Lectureship in Materials Science.

Congratulations to Juyoung Leem, from the University of Illinois at UrbanaChampaign, for winning the Arthur Nowick Graduate Student Award.

These highlights and more from the 2019 MRS Fall Meeting are available through MRS TV (mrs.org/mrs-tv) video capture, as well as news coverage in MRS Meeting Scene ${ }^{\circledR}$ (mrsmeetingscene.org).

Additional Meeting information can be accessed at mrs.org/fall2019.

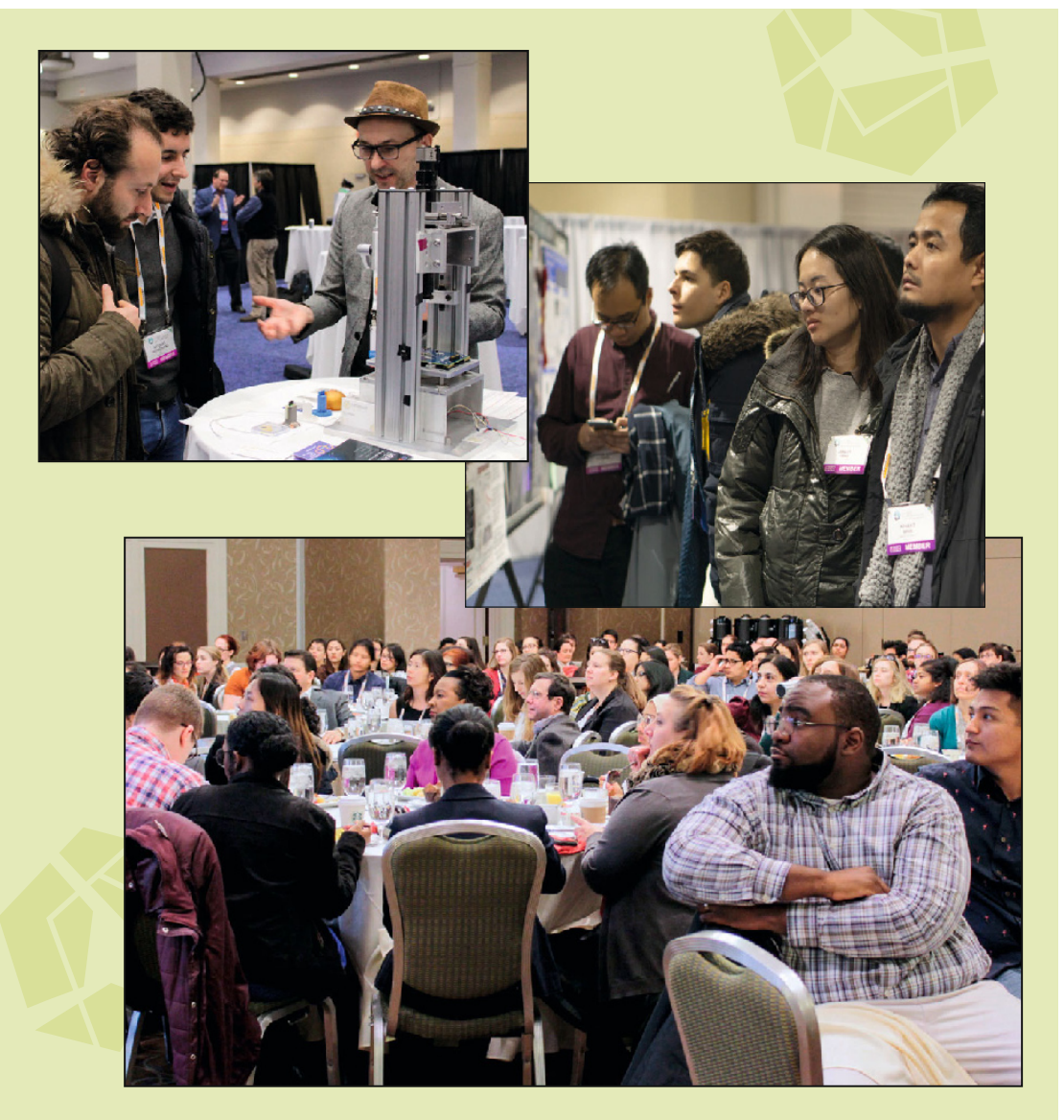

\section{Thank you! 2019 Materials Research Society Fall Meeting Symposium Support}

\section{PLATINUM}

IBM Q, IBM Research

The Kavli Foundation

\section{GOLD}

AIXTRON SE

Carl Zeiss Microscopy $\mathrm{GmbH}$

GVD Corporation

Hunt

Journal of Energy Chemistry

Dalian Institute of Chemical Physics

Lam Research Corporation

Raith America, Inc.

\section{SILVER}

2D Crystal Consortium - Materials Innovation Platform (2DCC-MIP)

Boston University Department of Mechanical Engineering

BUnano - BU Nanotechnology Innovation Center

Pacific Northwest National Laboratory Photon etc.

Seki Diamond Systems, Cornes Technologies

Specialty Coating Systems

TCL Research

ZEISS

\section{BRONZE}

1-Material Inc.

2D Materials | IOP Publishing

Accurion Inc.

ACS Applied Materials \& Interfaces |

ACS Publications

ACS Nano | ACS Publications

ACS Photonics | ACS Publications

Angstrom Engineering Inc.

Applied Diamond Inc.

Boston University Division of

Materials Science \& Engineering

Bruker

Citrine Informatics

DiamFab

ESIEE Paris - CCIR Paris lle-de-France

European Union's Horizon 2020

Research and Innovation programme under Grant Agreement No. 800926 (FET-OPEN-HyPhOE)

Forschungszentrum Jülich $\mathrm{GmbH}$

Fraunhofer USA - Center for Coatings and Diamond Technologies

GMW Associates

Graphenea Semiconductor SLU
J.A. Woollam Company, Inc. Journal of Materials Chemistry C | Royal Society of Chemistry Kleindiek Nanotechnik GmbH Los Alamos National Laboratory Center for Integrated

Nanotechnologies

Machine Learning: Science and Technology | IOP Publishing

Matter | Cell Press

Matter \& Patterns | Cell Press

Matter \& Trends in Chemistry | Cell Press

MilliporeSigma

Modelling and Simulation in Materials

Science and Engineering | IOP Publishing

Molecular Vista, Inc.

Morgan Advanced Materials

$M S D E$ | Royal Society of Chemistry

MTI Corporation

Multifunctional Materials | IOP Publishing

National Renewable Energy Laboratory

Organic Electronics | Elsevier

ORSAY PHYSICS
Oxford Instruments Asylum Research Inc.

Pacific Northwest National Laboratory

PhDTalent

Physical Electronics

Plasmability LLC

Protochips Inc.

Quantum Science and Technology | IOP Publishing

Raith America, Inc.

Rigaku

Royal Society of Chemistry

RWD Life Science

Scienta Omicron, Inc.

Semilab USA LLC

Sinton Instruments

SunMoon Materials Co.

TA Instruments

Ted Pella, Inc.

Thermo Fisher Scientific

Thermo-Calc Software Inc.

Tokyo Chemical Industry Co., Ltd

TOYO Corporation

Waterloo Institute for

Nanotechnology 